\section{Ifo 23}

VP-16 PLUS IFOSFAMIDE PLUS CISPLATIN (VIP) IN REFRACTORY GERM CELI TUMORS

P. J. Loehrer, S. D. Williams, and L. H. Einhorn

Forty-eight evaluable male patients with germ cell tumors (GCT) failing to be cured with first line therapy were treated with VP-16 (100 mg/m $\left.\mathrm{m}^{2}\right)$, ifosfamide $\left(1.2 \mathrm{mg} / \mathrm{m}^{2}\right)$, and cisplatin $\left(20 \mathrm{mg} / \mathrm{m}^{2}\right)$ a 11 given daily for five consecutive days every three weeks. All patients either achieved an unresectable partial remission as their best response to induction chemotherapy (Group A), relapsed from complete remission $\leq 2$ months after induction therapy (Group B), or had previously received cisplatin plus vp16 as previous salvage therapy (Group C). In our experience, these three groups represented patients in whom conventional salvage therapy with cisplatin pIus VP-16 alone rarely produced durable complete remissions. Nine (19\%) had extragonada1 GCT and $37(77 \%)$ had advanced disease. Twenty-three $(48 \%)$ of the patients had $\geq 2$ prior treatment regimens. Sixteen of 48 (33\%) achieved a complete remission (CR) to VIP alone or following surgical excision of residual disease. Six of $22(27 \%), 3$ of $7(43 \%)$ and $7 / 19(37 \%)$ of patients from groups A, B, and $C$ respectively attained a $C R$. The median survival time of all patients was 7 months (range 0-28+) with 7 patients remaining continuously free of disease ( 4 patients $>1$ year). Myelosuppression was significant with median WBC nadir of $900 / \mathrm{mm}^{2}$ and platelet nadir of $24,000 / \mathrm{mm}^{2}$. Fourteen $(26 \%)$ had granulocytopenic fever and $15 \%$ deve1oped renal insufficiency. VIP combination chemotherapy demonstrates activity in this highly unfavorable population of patients with germ cell tumors. These results compare favorably to our previous experience in similar patients treated with cisplatin plus VP-16 alone suggesting an important role for ifosfamide in this disease. Further studies with VIP as initial salvage therapy for patients with GCT are under way.

Indiana University, Room N582, 1100 W. Michigan Street, Indianapolis, IN 46223

\section{Ifo 24}

IFOSFAMIDE/MESNA IN COMBINATION CHEMOTHERAPY OF METASTATIC NONSEMINOMATOUS TESTICULAR CANCER AND SEMINOMAS

M.E. Scheulen, N. Niederle, K. Höffken, J. Schütte, S. Seeber and C.G. Schmidt

Although the prognosis of metastatic nonseminomatous test $i-$ cular cancer has been dramatically improved during the past decades due to the development of combination chemotherapy, the search for new chemotherapeutic alternatives for salvage therapy has become one of the major challenges.

Since 1977 we have investigated on the cytotoxic efficacy of ifosfamide atone (60mg $/ \mathrm{kg} /$ day iv, days $1-5, \mathrm{q} 21-28$ days) or in combination with etoposide $(40 \mathrm{mg} / \mathrm{kg} / \mathrm{day}$ ifosfamide $i v$, days $1-5,120 \mathrm{mg} / \mathrm{m}^{2} /$ day etoposide $i v$, days 1,3 , and $5, q$ 21-28 days! in 150 patients with metastatic nonseminomatous testicular cancer resistant against vinblastine, bleomycin, adriamycin, and cisolatinum.

Response rates - including minor resnonses - were $33 \%$ for ifosfamide (CR + PR 19\%) and $4.6 \%$ for i fosfamide/etoposide (CR $+P R \quad 30 \%$, respectively. The incidence of urinary tract complications, the dose limiting toxic side effect of ifosfamide, could be effectively reduced from $27 \%$ to $4 \%$ by coadministration of mesna $(12 \mathrm{mg} / \mathrm{kg}$ iv, $0, a$, and $8 \mathrm{~h}$ after ifosfamide).

Ifosfamide proved to be one of the most potent cytotoxic drugs in the primary treatment of metastatic seminomas, as well. Complete responses could be achieved in $16 / 17$ patients $(94 \%)$ by ifosfarmide, ifosfamide/etoposide or ifosfami$\mathrm{de} / \mathrm{cisplatinum}\left(40 \mathrm{mg} / \mathrm{kg} /\right.$ day ifosfamide iv, days $1-5,20 \mathrm{mg} / \mathrm{m}^{2}$ / day cisplatinum iv, days $1-5, q 21-28$ days). Presently, a 1 complete responders have no evidence of disease at a medium follow up of 28 months.

In conclusion, ifosfamide/mesna - one of the most active cytotoxic agents in metastatic testicular cancer - may not on$1 y$ be included into effective combination chemotherapy regimens for salvage therapy but also in the first line treatment of this malignant disease.

Dept. Intern. Med. (Cancer Research), West German Tumor Center, Hufelandstr. 55, D-4300 Essen ?

\section{Ifo 25}

IFOSFAMIDE IN TREATMENT OF LUNG CANCER P. Drings

Ifosfamide is used as single drug of in combination with other cytostatic agents in the treatment of lung cancer. Different schedules of single agent therapy- 24 hours infusion, bolus $\left(5 \mathrm{mg} / \mathrm{m}^{2}\right)$ infusion over 30 minutes or treatment with 7,0 to 2,0 $\mathrm{g} / \mathrm{m}^{2}$ days $1-5$ are performed.

Small cell lung cancer (SCLC):

Different study groups reported response rates after ifosfamide alone of $5-34^{\circ}$ even in pretreated patients. Ifosfamide was introduced into different combinations. In a randomized study, including 305 patients, alternating chemotherapy with etopaside, ifosfamide, vindesine (VPIV), adriamycin, cisplatinum, vincristine (APO), and cyclophosphamide, methotrexate, CCNU [CMCC] was compared to standard treatment alternating therapy resulted in higher response rate, and a longer median survival time. Regarding toxicity, VPIV was similiar to ACO, whereas APO and CMCC had more side effects.

In a 2nd randomized study 142 patients were thated either with ifosfamideletoposide (IVP) or with cisplatinum/etoposide (PVP). Both regimes were comparable regarding to reserved in patients with PVP.

Non-small cell lung cancer (NSCLC):

With response rates from 10 to $39 \frac{0}{2}$ ifosfamide is one of the most active drugs in NSCLC, too. In 2 consecutive phase $/ 1$ studies including 162 patients the cytostatic effect of ifosfamide in combination with cisplatinum or etoposide was investigated. An objectively measurable tumor regression was achieved in $37 \%$ of the patients including 5 complete and 45 partial remissions. The median survival time for all 162 patients was calculated with 8.5 months. In both studies responders survived significantly longer than nonresponders ( 13 versus 5 months). Because of the better tolerability the combination ifosfamide/etoposide is superior to some cisplatinum combino tion (especially high dose cisplatinum). Therefore we would prefer this combination in the treatment of NSCLC.

Klinik für Thoraxerkrankungen der LVA Baden, Amalienstrasse $5, D^{-}=6900$ Heidelberg 1

\section{Ifo 26}

BIOAVAILABILITY OF IFOSFAMIDE IN PATIENTS WITH BRONCHIAI CARCINOMA.

T.Cerny, J Margison, I. Thatcher, P.M.Wilkinson.

The aim of this study was to assess the biovailability of Ifosfamide (I).

Within patient pharmacokinetics of $(I)$ were determined following the administration of $1 \mathrm{~g}$ and $2 \mathrm{~g} \mathrm{p.o.} \mathrm{and} \mathrm{i} . \mathrm{v}$. in seven patients with bronchial carcinoma. Serial serum and urine samples were collected during the first 48 hours after administration and concentrations of (I) were assayed by HPLC using a method developed in this laboratory. The AUC $\left(\mu \mathrm{g} \cdot \mathrm{L} \cdot \mathrm{h}^{-1}\right)$ for both the 1 and $2 \mathrm{~g}$ doses were the same following p.o. and i.v. administration indicating 100\% bioavailability (Table 1). The i.v. serum concentration/time curve exhibited a biphasic decay with a terminal half life of $5.92 \mathrm{~h}$ (SD 1.15, SEM 0.47) for $1 \mathrm{~g}$ and 5.29 (SD 0.73, SEM 0.27) for $2 \mathrm{~g}$. Drug clearance was similar for both methods of administration. We conclude that up to $2 \mathrm{~g}$ of (I) p.o. is an alternative route of administration and makes out patient use a possibility.

Table 1. Bioavailability of Ifosfamide

\begin{tabular}{|c|c|c|c|c|}
\hline Dose $g$. & \multicolumn{2}{|c|}{1} & \multicolumn{2}{|c|}{2} \\
\hline & $\mathrm{p} . \mathrm{o}^{-}$ & i.v. & p.o. & i.v. \\
\hline$\mu g \cdot I \cdot h^{-1}$ & 266.3 & 294.2 & 511.8 & 478.2 \\
\hline$S D$ & 12.02 & 29.82 & 83.97 & 34.86 \\
\hline SEM & 4.90 & 12.17 & 31.73 & 13.17 \\
\hline
\end{tabular}

AUC $=$ Area under the curve

$\mathrm{SD}=$ Standard deviation

SEM = Standard error of the mean.

Departments of Medical Oncology and Clinical Pharmacology, Christie Hospital \& folt Radium Institute, wilmslow Road Manchester UK 